\title{
FERMION DAMPING RATE EFFECTS IN COLD DENSE MATTER ${ }^{a b}$
}

\author{
Cristina Manuel \\ Theory Division, CERN, CH-1211 Geneva 23, Switzerland \\ E-mail:Cristina.Manuel@cern.ch
}

\begin{abstract}
We review the non-Fermi or marginal liquid behavior of a relativistic QED plasma. In this medium a quasiparticle has a damping rate that depends linearly on the distance between its energy and the Fermi surface. We stress that this dependence is due to the long-range character of the magnetic interactions in the medium. Finally, we study how the quark damping rate modifies the gap equation of color superconductivity, reducing the value of the gap at the Fermi surface.
\end{abstract}

\section{Introduction}

There is an increasing interest in studying how matter behaves at very high density. While high density effects in non-relativistic systems have been studied thoroughly in the past, the same does not hold true for relativistic ultradegenerate plasmas. Relativistic effects cannot be avoided if the chemical potential $\mu$ of the system is much larger than the mass of the particles that form the medium. This situation certainly occurs in the interior of neutron stars. The astrophysical scenario is the natural domain of application of the physics of ultradegenerate relativistic plasmas.

Electromagnetic plasmas behave in a drastically different way in their nonrelativistic and ultrarelativistic limits. This is so because the magnetic interactions are suppressed in the non-relativistic limit by powers of $v^{2} / c^{2}$, where $v$ is the typical velocity of the particles in the plasma, and $c$ is the velocity of light. Electric and magnetic interactions behave in a very different way in a plasma. In the medium, static electric fields are completely screened. This is the wellknown Debye screening phenomenon, also known as Thomas-Fermi screening for ultradegenerate plasmas. But magnetic interactions are only weakly dynamically screened, through Landau damping. Thus, while electric interactions are short-ranged, magnetic interactions are long-ranged. This fact has several relevant consequences for ultradegenerate plasmas and makes the relativistic and non-relativistic phases of the plasma to look completely different.

In this talk we will discuss how the long-range character of magnetic interactions affects the lifetime of a quasiparticle in the medium. This is based on work done in collaboration with Michel Le Bellact. We will then see how

${ }^{a}$ Talk given at "Strong and Electroweak Matter 2000", Marseille, June 2000.

${ }^{b}$ CERN-TH/2000-277 
the fermion lifetime effects also correct the value of the gap of color superconductivity $\mathrm{B}$.

\section{Lifetime of a quasiparticle and non-Fermi liquid behavior of the relativistic plasmas}

One of the central concepts in a plasma is that of a quasiparticle. A particle immersed in a medium modifies its propagation properties by interacting with the surrounding medium. In field theoretical language, we would say that the particle is "dressed" by a self-energy cloud. In the ultradegenerate plasma, the relevant degrees of freedom are those of quasiparticles or quasiholes (absences of particles in the Fermi sea) living close to the Fermi surface. Because of the exclusion principle, quasiparticles/quasiholes can only live if they are outside/inside the Fermi sea. These excitations tend to lower their energy by undergoing collisions with the particles in the Fermi sea. They decay, and thus have a finite lifetime. The concept of quasiparticle, however, only makes sense if its lifetime is long enough, or in other words, if its damping rate is much smaller than its energy.

If the interactions in the system are repulsive and short-ranged, some of the propagation properties of the quasiparticles can be deduced on general grounds. In that case, Luttinger's theorem 6 states the energy dependence of the damping rate of a quasiparticle that lives close to the Fermi surface. The damping rate can be obtained either by computing the imaginary part of the fermion self-energy or, alternatively, by computing the decay rate

$$
\begin{aligned}
\Gamma(E) & =\frac{1}{E} \int \frac{\mathrm{d}^{3} p^{\prime}}{(2 \pi)^{3}} \frac{\left(1-\Theta\left(\mu-E_{p^{\prime}}\right)\right)}{2 E_{p^{\prime}}} \int \frac{\mathrm{d}^{3} k}{(2 \pi)^{3}} \frac{\Theta\left(\mu-E_{k}\right)}{2 E_{k}} \\
& \times \int \frac{\mathrm{d}^{3} k^{\prime}}{(2 \pi)^{3}} \frac{\left(1-\Theta\left(\mu-E_{k^{\prime}}\right)\right)}{2 E_{k^{\prime}}}(2 \pi)^{4} \delta^{(4)}\left(P+K-P^{\prime}-K^{\prime}\right)|\mathcal{M}|^{2},
\end{aligned}
$$

where $|\mathcal{M}|^{2}$ is the scattering matrix element squared, and $\Theta$ is the step function. The above decay rate represents the interaction of the quasiparticle with one fermion inside the Fermi sea with energy $E_{k}$. As a result, two new particles appear, with energies $E_{k^{\prime}}$ and $E_{p^{\prime}}$, which are outside the Fermi sea. If the interaction is repulsive and short-ranged, and for $E-\mu \ll \mu$, one can take $|\mathcal{M}|^{2}$ with the value of the fermion energies at $\mu$. Then, one can deduce that $\Gamma(E) \propto(E-\mu)^{2}$, only using the phase-space restrictions of fermion-fermion scattering.

The damping rate can also be obtained from the imaginary part of the fermion self-energy, and the computation agrees with that obtained from Eq. (11). Since the real and imaginary parts of the self-energy corrections are related 
by dispersion relations, Luttinger's theorem implies that the leading order behavior of the real part of the self-energy is $\propto|E-\mu|$. For weakly coupled systems, the dispersion relations of the quasiparticles (or quasiholes) are not drastically affected by medium effects.

For systems with long-range interactions, it is not possible to make the previous general statements, as in general the integral in Eq. (1i) will depend on the form of the interaction, and in general, Luttinger's theorem will be violated. This is actually what happens in relativistic QED plasmas, due to the long-range character of the magnetic interactions. A closer look into the decay rate Eq. (11) in a relativistic plasma shows that it is dominated by scattering in the forward or collinear direction, mediated by a soft Landau damped magnetic photon. The momentum of the photon in the process is space-like, so the fermion damping fate would vanish in the absence of Landau damping. In particular, one finds 0.5

$$
\operatorname{Im} \Sigma_{+}(E, p) \sim \frac{e^{2}}{24 \pi}|E-\mu|,
$$

where $e$ is the electromagnetic coupling constant. The real part of the selfenergy can be computed from the imaginary part, using a dispersion relation. One then finds 6 国

$$
\operatorname{Re} \Sigma_{+}(E, p) \sim \frac{e^{2}}{12 \pi^{2}}(E-\mu) \ln \frac{M}{|E-\mu|}+\mathcal{O}((E-\mu)) .
$$

The wavefunction renormalization factor $Z$ can then be equally computed from the above values. One finds

$$
Z^{-1} \sim 1-\frac{e^{2}}{12 \pi^{2}} \ln \frac{M}{|E-\mu|} .
$$

Thus, in the limit $E \rightarrow \mu$, the fermion propagator vanishes, instead of showing the typical step discontinuity associated to the existence of a Fermi surface 7. This is an anomalous behavior for a typical Fermi liquid. Its origin is the long-range character of the magnetic interactions in the relativistic system.

\section{Color superconductivity at weak coupling}

QCD at very high baryonic density behaves as a color superconductor 6 . This is a consequence of Cooper's theorem, as any attractive interaction occurring close to the Fermi surface makes the system unstable to the formation of particle pairing. In QCD the attractive interaction is provided by one-gluon exchange in a color antisymmetric $\overline{3}$ channel. 
In the weak coupling limit the value of the gap can be computed in perturbation theory. The condensation process is dominated by the exchange of very soft magnetic gluons, which are dynamically screened by Landau damping. The Meissnef effect is a subleading effect in the gap equation. At leading

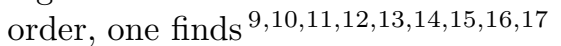

$$
\phi_{0} \sim 2 \frac{b_{0}}{g^{5}} \mu \exp \left(-\frac{3 \pi^{2}}{\sqrt{2} g}\right)[1+\mathcal{O}(g)], \quad b_{0}=256 \pi^{4}\left(\frac{2}{N_{f}}\right)^{5 / 2} b_{0}^{\prime},
$$

where $g$ is the gauge coupling constant, $N_{f}$ is the number of quark flavors, and $b_{0}^{\prime}$ is a constant of order one. The dependence on the coupling constant of the gap is quite different from the one that arises in a system with short-range interactions.

It is possible to compute next to leading order corrections to Eq. (5) by introducing one-loop corrections in the quark propagators of the gap equation. Here, we will mainly concentrate on studying how the quark damping rate affects the value of the gap close to the Fermi surface. At leading order one finds a modified gap equation 3

$$
\phi_{k}=\frac{g^{2}}{36 \pi^{2}} \int_{0}^{\infty} d(q-\mu)\left[\ln \left(\frac{\mu^{2} b^{2}}{\left|\epsilon_{q}^{2}-\epsilon_{k}^{2}\right|}\right)\right] \frac{\phi_{q}}{\epsilon_{q}} \frac{2}{\pi} \arctan \left(\frac{\epsilon_{q}}{\Gamma_{q}}\right),
$$

where $b=b_{0} / g^{5}, \epsilon_{q}=\sqrt{(q-\mu)^{2}+\phi_{q}^{2}}$, and $\Gamma_{q}$ is the quark damping rate. The most relevant effect of the damping rate is introducing a physical ultraviolet cutoff in the gap equation: when the ratio $\epsilon_{q} / \Gamma_{q}$ starts to be small, the integrand in Eq. (6) tends to zero. This situation actually occurs for quarks that are far away from the Fermi surface. As expected, the condensation process only occurs close to the Fermi surface.

For a leading order computation of the gap at the Fermi surface one can take the value of $\Gamma_{q}$ in the normal phase of the system (that is, using Eq. (2), replacing $e^{2}$ by $\frac{4}{3} g^{2}$ ). This is so because the one-loop fermion self-energy in the normal phase differs from that in the superconducting phase by, at most, values of the order of the squared of the condensate. Also, the Meissner effect in the gluon propagator to arrive at the value of $\Gamma_{q}$ is a subleading effect. The dominant scattering processes are those occurring in the forward direction, and these processes are dominated by soft Landau-damped color magnetic interactions, exactly as it happens in the gap equation. To leading order one finds 3

$$
\phi_{0}^{\text {damp }} \sim 2 \frac{b_{0}}{g^{5}} \mu \exp \left(-\frac{\pi}{2 \bar{g}_{e f f}}\right)
$$


where $\bar{g}_{\text {eff }}^{2}=\bar{g}^{2}\left(1-2 \bar{g}^{2}\right)$, and $\bar{g}=g / 3 \sqrt{2} \pi$. The value of the gap at the Fermi surface is then reduced. This can be understood in very intuitive terms. The fact that the quarks decay limits their efficiency to condense. The decay of the quasiparticles should also affect the critical temperature of transition to the normal phase of the system computed in Refs. 11 . 12 . 6 . 127 .

The fermion damping rate effects represent a correction of order $g^{2}$ to the leading order value Eq. (5). It is worth emphasizing that this is not the complete next-to-leading order correction, which it should be possible to compute using the Schwinger-Dyson equations.

\section{Acknowledgements}

I would like to thank H. Ren for very useful discussions on the non-Fermi liquid behavior of the QED plasmas. My gratitude also goes to the organizers of this nice meeting. Financial support from a Marie Curie EC Grant (HPMFCT-1999-00391) is acknowledged.

\section{References}

1. M. Le Bellac and C. Manuel, Phys. Rev. D 55, 3215 (1997).

2. C. Manuel, Phys. Rev. D 62, 76009 (2000).

3. C. Manuel, hep-ph/0006106, to be published in Phys. Rev. D.

4. J. M. Luttinger, Phys. Rev. 121, 942 (1961).

5. B. Vanderheyden and J. Ollitrault, Phys. Rev. D 56, 5108 (1997).

6. W. E. Brown, J. T. Liu and H. Ren, Phys. Rev. D 62, 054013 (2000).

7. T. Holstein, R. Norton and P. Pincus, Phys. Rev. B 8, 264 (1973). M. Yu. Reizer, Phys. Rev. B 40, 11571 (1989); S. Chakravarty, R. E. Norton, and O. F. Syljuasen, Phys. Rev. Lett. 74, 1423 (1995).

8. See K. Rajagopal's talk in these Proceedings for a general review on color superconductivity.

9. D. T. Son, Phys. Rev. D 59, 094019 (1999).

10. T. Schäfer and F. Wilczek, Phys. Rev. D 60, 114033 (1999).

11. R. D. Pisarski and D. H. Rischke, Phys. Rev. D 61, 051501 (2000).

12. R. D. Pisarski and D. H. Rischke, Phys. Rev. D 61, 074017 (2000).

13. D. K. Hong, Phys. Lett. B 473, 118 (2000).

14. D. K. Hong, V. A. Miransky, I. A. Shovkovy and L. C. Wijewardhana, Phys. Rev. D 61, 056001 (2000).

15. N. Evans, J. Hormuzdiar, S. D. Hsu and M. Schwetz, Nucl. Phys. B 581, 391 (2000).

16. W. E. Brown, J. T. Liu and H. Ren, Phys. Rev. D 61, 114012 (2000).

17. W. E. Brown, J. T. Liu and H. Ren, Phys. Rev. D 62, 054016 (2000). 